\title{
Development of Isocratic RP-HPLC Method for Separation and Quantification of L-Citrulline and L-Arginine in Watermelons
}

\author{
Rasdin Ridwan $\left(\mathrm{D},{ }^{1}\right.$ Hairil Rashmizal Abdul Razak, ${ }^{2}$ \\ Mohd Ilham Adenan, ${ }^{3,4}$ and Wan Mazlina Md Saad $\mathbb{D}^{1}$ \\ ${ }^{1}$ Centre of Medical Laboratory Technology, Faculty of Health Sciences, Universiti Teknologi MARA, Puncak Alam Campus, \\ 42300 Bandar Puncak Alam, Selangor, Malaysia \\ ${ }^{2}$ Centre of Medical Imaging, Faculty of Health Sciences, Universiti Teknologi MARA, Puncak Alam Campus, \\ 42300 Bandar Puncak Alam, Selangor, Malaysia \\ ${ }^{3}$ Faculty of Applied Sciences, Universiti Teknologi MARA, 40450 Shah Alam, Selangor, Malaysia \\ ${ }^{4}$ Atta-ur-Rahman Institute for Natural Product Discovery, Level 9, Bangunan FF3, Universiti Teknologi MARA, Puncak Alam Campus, \\ 42300 Bandar Puncak Alam, Selangor, Malaysia
}

Correspondence should be addressed to Wan Mazlina Md Saad; mazlinasaad14@gmail.com

Received 30 December 2017; Revised 25 February 2018; Accepted 12 March 2018; Published 2 May 2018

Academic Editor: Günther K. Bonn

Copyright (c) 2018 Rasdin Ridwan et al. This is an open access article distributed under the Creative Commons Attribution License, which permits unrestricted use, distribution, and reproduction in any medium, provided the original work is properly cited.

\begin{abstract}
Watermelons (Citrullus lanatus) are known to have sufficient amino acid content. In this study, watermelons grown and consumed in Malaysia were investigated for their amino acid content, L-citrulline and L-arginine, by the isocratic RP-HPLC method. Flesh and rind watermelons were juiced, and freeze-dried samples were used for separation and quantification of L-citrulline and $\mathrm{L}$-arginine. Three different mobile phases, $0.7 \% \mathrm{H}_{3} \mathrm{P0}_{4}, 0.1 \% \mathrm{H}_{3} \mathrm{P0}_{4}$, and $0.7 \% \mathrm{H}_{3} \mathrm{P0}_{4}$ : $\mathrm{ACN}(90: 10)$, were tested on two different columns using Zorbax Eclipse XDB-C ${ }_{18}$ and Gemini $\mathrm{C}_{18}$ with a flow rate of $0.5 \mathrm{~mL} / \mathrm{min}$ and a detection wavelength at $195 \mathrm{~nm}$. Efficient separation with reproducible resolution of $\mathrm{L}$-citrulline and $\mathrm{L}$-arginine was achieved using $0.1 \% \mathrm{H}_{3} \mathrm{P}_{4}$ on the $\mathrm{Gemini}_{18}$ column. The method was validated and good linearity of $\mathrm{L}$-citrulline and L-arginine was obtained with $R^{2}=0.9956, y=0.1664 x+2.4142$ and $R^{2}=0.9912, y=0.4100 x+3.4850$, respectively. L-citrulline content showed the highest concentration in red watermelon of flesh and rind juice extract $(43.81 \mathrm{mg} / \mathrm{g}$ and $45.02 \mathrm{mg} / \mathrm{g})$, whereas L-arginine concentration was lower than L-citrulline, ranging from 3.39 to $11.14 \mathrm{mg} / \mathrm{g}$. The isocratic RP-HPLC method with $0.1 \% \mathrm{H}_{3} \mathrm{P}_{4}$ on the Gemini $\mathrm{C}_{18}$ column proved to be efficient for separation and quantification of L-citrulline and L-arginine in watermelons.
\end{abstract}

\section{Introduction}

Citrullus lanatus (Thunb.) Matsum. and Nakai, commonly known as watermelon, is a nonseasonal fruit which is cultivated abundantly in Malaysia and other tropical regions [1]. It belongs to the Cucurbitaceae plant family, which originated from the African Kalahari Desert [1]. Watermelons have high content of phytonutrients and are rich in dietary antioxidants such as carotenoids (lycopene and $\beta$-carotene), polyphenolics, ascorbic acid, and significant amino acids [2] Watermelons are usually consumed by juicing the flesh, beneficial in the prevention and improvement of health problems, such as cardiovascular diseases, erectile dysfunction, hypertension, and cancers [3]. Figueroa et al. [4] demonstrated that watermelon juice supplementation improves aortic hemodynamics by reducing the reflected wave amplitude in prehypertensive individuals. A study by Poduri et al. [5] reported that watermelon attenuated hypercholesterolemiainduced atherosclerosis in mice. Commercial watermelon juices provide enormous marketing potential and nutritious drinks for individuals to maintain a healthy lifestyle.

Amino acids, particularly L-citrulline and L-arginine, are regarded as major types of phytonutrients present in watermelons which may contribute to their reputed and diversified health benefits [6]. L-citrulline, $\mathrm{C}_{6} \mathrm{H}_{13} \mathrm{~N}_{3} \mathrm{O}_{3}$ (IUPAC name: 2-amino-5-(carbamoylamino)pentanoic acid) (Figure 1), is a nonessential amino acid firstly identified from watermelon, Citrullus vulgaris Schrad. [7, 8]. L-citrulline is a physiological 
<smiles>NC(=O)NCCC[C@H](N)C(=O)O</smiles>

FIgURE 1: Molecular structure of L-citrulline $(175.2 \mathrm{~g} / \mathrm{mol})$.<smiles>N=C(N)NCCC[C@H](N)C(=O)O</smiles>

FiguRE 2: Molecular structure of L-arginine (174.2 g/mol).

endogenous amino acid to most living systems involved in protein metabolism and removal of excess metabolic ammonia [9]. It serves as a precursor for $\mathrm{L}$-arginine and product of nitric oxide (NO) cycle [10]. L-arginine, $\mathrm{C}_{6} \mathrm{H}_{14} \mathrm{~N}_{4} \mathrm{O}_{2}$ (IUPAC name: (S)-2-amino-5-guanidinopentanoic acid) (Figure 2), is a semiessential and free form physiological amino acid that functions as one of 20 building block proteins for biological processes such as cell division, ammonia removal, wound healing, and hormone release $[7,8]$. Wu et al. [11] demonstrated that supplementation of L-citrulline and Larginine from watermelon juice improved serum levels of $\mathrm{NO}$ metabolites and aortic endothelial-mediated vasodilation in diabetic rats.

L-citrulline and L-arginine are present in all parts of watermelon fruits including flesh, rind, and seed [7]. A study done by Rimando and Perkins-Veazie [12] reported that the rind of red watermelon and yellow watermelon contains more L-citrulline at a concentration ranging from 15.6 to $29.4 \mathrm{mg} / \mathrm{g}$ than flesh, $7.9-28.5 \mathrm{mg} / \mathrm{g}$. Similar to the above finding, Jayaprakasha et al. [13] reported that rinds of Citrullus vulgaris varieties such as petite treat and jamboree watermelon and also yellow crimson watermelon contained slightly higher L-citrulline ranging from 13.95 to $28.46 \mathrm{mg} / \mathrm{g}$ than flesh, $11.25-16.73 \mathrm{mg} / \mathrm{g}$. These findings suggested that watermelon rind has an abundance of L-citrulline content in comparison to its content in flesh.

Analyses of L-citrulline and L-arginine were routinely conducted using capillary electrophoresis and quantification by a spectrophotometric method; however, the method is less sensitive, leading to discrepancies in the outcomes [6]. L-citrulline and L-arginine are polar, nonvolatile, and devoid of chromophores; thus analysis by reverse-phase high performance liquid chromatography (RP-HPLC) commonly employed a derivatization method using pre- or postcolumn derivatization [14-17]. Jayaprakasha et al. [13] stated that precolumn derivatization such as orthophthalaldehyde (OPA), naphthalene-2,3-dicarboxaldehyde, or 4-dimethylaminoazobenzene- $4^{\prime}$-sulfonyl chloride (dabsyl chloride) was able to provide accurate and stable chromatography baseline, but the reactions were unstable and affected by the sample matrix [18]. Postcolumn derivatization by ninhydrin is tedious due to long analysis time up to 72 hours and instability of derivatization reagents that may cause poor compound recovery [19]. Analysis of underivatized L-citrulline and Larginine is warranted for rapid and effective quantification of these compounds. Given that no amino acids content of Lcitrulline and L-arginine in Malaysia watermelons has been reported so far, we have developed an isocratic RP-HPLC method for separation and quantification of L-citrulline and $\mathrm{L}$-arginine in watermelons.

\section{Materials and Methods}

2.1. Chemicals and Reagents. L-citrulline (purity $\geq 99 \%$ ) and L-arginine (purity $\geq 98 \%$ ) standard were purchased from Sigma-Aldrich (St. Louis, MO, USA). Methanol and acetonitrile of HPLC grade were purchased from Merck (Germany). Phosphoric acid (purity $\geq 85 \%$ ) was purchased from Sigma-Aldrich (St. Louis, MO, USA). Deionized water was prepared using ultrapure water purifier system (Elgastat, Bucks, UK).

2.2. Instrumentation. The isocratic RP-HPLC method was carried out using Thermo Scientific ${ }^{\mathrm{TM}}$ Dionex-UltiMate ${ }^{\mathrm{TM}}$ 3000 HPLC system equipped with solvent reservoirs, LPG3400SD pump, WPS-3000 autosampler injector, TCC-3000 column oven, and DAD-3000 ultraviolet-visible (UV-Vis) diode array detector module operated at four wavelengths per analysis. Chromeleon data software (Version 7) was used for data analysis.

2.3. Sample Preparation. Citrullus lanatus (Thunb.) Matsum. \& Nakai of red watermelon and yellow crimson watermelon was obtained from Selangor Fruit Valley, Selangor. Seeds were removed manually and the edible part was cut into cubes. Watermelon flesh and rind were juiced and frozen at $-80^{\circ} \mathrm{C}$ for at least 2 days. The frozen juices were put in a freeze-drier (Labconco, USA) for 4 days until completely dried. The dried juice powders were kept at $-20^{\circ} \mathrm{C}$. For the analysis, samples were prepared in the form of juice extract and methanol extract. Juice extract was prepared directly by dissolving the dried juice powder in $\mathrm{dH}_{2} \mathrm{O}$. For methanol extract, a known quantity of dried juice powders was extracted with $30 \mathrm{~mL}$ of $\mathrm{MeOH}$ and $1 \mathrm{~mL}$ of $1 \mathrm{~N} \mathrm{HCl}$, vortexed, and sonicated for 30 minutes. The samples were macerated by cold maceration for a period of 72 hours in an orbital shaker. Methanol extracts were then filtered using Whatman filter paper. The residues were reextracted twice using fresh solvent and the three methanol extracts were pooled. The obtained methanol extracts were evaporated to dryness using a rotary vacuum evaporator at $60^{\circ} \mathrm{C}$ and stored at $4^{\circ} \mathrm{C}$ until analysis.

\subsection{Isocratic RP-HPLC Analysis}

2.4.1. Standard Preparation Procedure. A stock solution of Lcitrulline and L-arginine was prepared individually in $\mathrm{dH}_{2} \mathrm{O}$ at $1 \mathrm{mg} / \mathrm{mL}$ and filtered through a $0.45 \mu \mathrm{m}$ syringe filter (Bioflow). A mixed standard solution was prepared by mixing an equal volume of each standard stock solution. A series of working standard solutions was prepared by diluting the stock solution with $\mathrm{dH}_{2} \mathrm{O}$ in the range of $0.1-1000 \mu \mathrm{g} / \mathrm{mL}$. 
TABLE 1: Selection of mobile phases for separation of mixed standard, L-citrulline, and L-arginine by isocratic RP-HPLC.

\begin{tabular}{lcc}
\hline Mobile phase & Ratio & Solution mixture (\%) \\
\hline $0.7 \% \mathrm{H}_{3} \mathrm{PO}_{4}$ & 100 & $0.7 \% \mathrm{H}_{3} \mathrm{P0}_{4}+99.3 \% \mathrm{dH}_{2} \mathrm{O}$ \\
$0.1 \% \mathrm{H}_{3} \mathrm{PO}_{4}$ & 100 & $0.1 \% \mathrm{H}_{3} \mathrm{P}_{4}+99.9 \% \mathrm{dH}_{2} \mathrm{O}$ \\
$0.7 \% \mathrm{H}_{3} \mathrm{PO}_{4}: \mathrm{ACN}$ & $90: 10$ & $\left(0.7 \% \mathrm{H}_{3} \mathrm{P0}_{4}+99.3 \% \mathrm{dH}_{2} \mathrm{O}\right)+$ \\
\end{tabular}

2.4.2. Sample Preparation Procedure. Juice extracts were prepared directly by dissolving the dried juices powder in $\mathrm{dH}_{2} \mathrm{O}$ at $5 \mathrm{mg} / \mathrm{mL}$. Crude methanol extracts were also dissolved in $\mathrm{dH}_{2} \mathrm{O}$ at $5 \mathrm{mg} / \mathrm{mL}$ and vortexed for 15 minutes. All extracts were filtered through $0.45 \mu \mathrm{m}$ filters and injected to isocratic RP-HPLC.

2.4.3. Chromatographic Analysis. Preliminarily, three different concentrations of ion-pair reagents, phosphoric acid $\left(\mathrm{H}_{3} \mathrm{PO}_{4}\right)$, or addition of acetonitrile $(\mathrm{ACN})$ as mobile phases, $0.7 \% \mathrm{H}_{3} \mathrm{PO}_{4}, 0.1 \% \mathrm{H}_{3} \mathrm{P0}_{4}$, and $0.7 \% \mathrm{H}_{3} \mathrm{PO}_{4}: \mathrm{ACN}(90: 10)$ (Table 1), were tested for separation and determination of Lcitrulline and L-arginine standard. The column temperature was fixed at room temperature and UV-Vis detection was performed at $195 \mathrm{~nm}$. The RP-HPLC columns [i.e., Zorbax Eclipse $\mathrm{XDB}-\mathrm{C}_{18}, 250 \mathrm{~mm} \times 4.6 \mathrm{~mm}, 80 \AA, 5 \mu \mathrm{m}$ (Phenomenex, Torrance, CA), and $\mathrm{Gemini}_{18}, 250 \times 4.6 \mathrm{~mm}$, $110 \AA, 3 \mu \mathrm{m}$ (Phenomenex, Torrance, CA)] were used. The analysis proceeded for quantification of both compounds, L-citrulline and L-arginine in watermelons juice extracts and methanol extracts using the chosen column and mobile phase: Gemini $\mathrm{C}_{18}$ eluted by $0.1 \% \mathrm{H}_{3} \mathrm{P0}_{4}$ with a flow rate of $0.5 \mathrm{~mL} / \mathrm{min}$ at $195 \mathrm{~nm}$. Chromeleon software was used for quantification of L-citrulline and L-arginine. The concentration of L-citrulline and L-arginine content was quantified based on the linear curve of standards. The content of compounds was expressed as milligrams per gram $(\mathrm{mg} / \mathrm{g})$ of sample extracts.

2.4.4. Method Validation. The validation of the isocratic RPHPLC method was performed for linearity of calibration curve, limit of detection (LOD), limit of quantification (LOQ), accuracy, and precision. The linearity of the isocratic RP-HPLC method for quantification of compounds was constructed using the concentration range of $0.1-1000 \mu \mathrm{g} / \mathrm{mL}$ for $\mathrm{L}$-citrulline and $0.1-500 \mu \mathrm{g} / \mathrm{mL}$ for $\mathrm{L}$-arginine. The regression equation was calculated in the form of $y=a x+b$, where $x$ is the concentration and $y$ is the peak area of compounds. Linearity was established by the coefficient of determination $\left(R^{2}\right)$. LOD and LOQ were measured based on signal-tonoise ratio $(\mathrm{S} / \mathrm{N})$ method. LOD is the lowest concentration of analyte that can be detected with signal-to-noise ratio of $3: 1$ and LOQ is the lowest concentration that can be quantified with acceptable precision and accuracy with signal-to-noise ratio of $10: 1$. S/N of 3 is considered acceptable for LOD, while LOQ is established at $\mathrm{S} / \mathrm{N}$ of 10 . Precision of the method was determined as percentage relative standard deviation (\%RSD) of peak area of intraday and interday analysis data. Intraday (three times in a day operation under the same conditions) and interday (three different days) studies were performed at three different concentrations (Level 1:20 $\mu \mathrm{g} / \mathrm{mL}$; Level 2: $60 \mu \mathrm{g} / \mathrm{mL}$; Level 3: $150 \mu \mathrm{g} / \mathrm{mL}$ ). The resulting peak area was used to calculate SD and the relative standard deviation (\%RSD). Accuracy of the method by recovery study was done by adding a known amount of reference standard solution (three concentrations) to test samples. The spiked extract solutions were injected three times, and the recovery was calculated with the value of detected versus added amounts.

\section{Results and Discussion}

3.1. Separation of L-Citrulline and L-Arginine by Isocratic RPHPLC Method. The initial isocratic RP-HPLC method for separation of mixed standard, L-citrulline, and L-arginine was performed using selected mobile phases according to previous literatures with slight modifications [8, 13, 20]. Interaction between mobile phase and stationary phases in isocratic RP-HPLC is important for the determination of solutes' retention time [21].

In this study, separation for determination of mixed standard, L-citrulline, and L-arginine was performed using a hydrophilic anionic ion-pairing reagent with different concentrations of phosphoric acid $\left(\mathrm{H}_{3} \mathrm{PO}_{4}\right)$ or addition of acetonitrile $(\mathrm{ACN})$ as mobile phases: $0.7 \% \mathrm{H}_{3} \mathrm{P0}_{4}, 0.1 \%$ $\mathrm{H}_{3} \mathrm{P}_{4}$, and $0.7 \% \mathrm{H}_{3} \mathrm{PO}_{4}: \mathrm{ACN}(90: 10)$. The mobile phase at the concentration of $0.7 \% \mathrm{H}_{3} \mathrm{PO}_{4}$ : ACN $(90: 10)$ resulted in L-citrulline and L-arginine were unretained and coeluted $(k$ value close to 0 ) as shown in Figure 3(a). The mixture of $0.7 \%$ $\mathrm{H}_{3} \mathrm{P0}_{4}$ : ACN $(90: 10)$ is highly hydrophilic, leading to rapid elution of L-citrulline and L-arginine with poor separation. Peaks of L-citrulline and L-arginine were slightly retained and partially separated using $0.7 \% \mathrm{H}_{3} \mathrm{P}_{4}$ (Figure $3(\mathrm{~b})$ ). However, optimum resolution was not achieved by $0.7 \%$ $\mathrm{H}_{3} \mathrm{PO}_{4}$ as $k$ value between $\mathrm{L}$-citrulline and L-arginine is close to 1 . The mobile phase of $0.1 \% \mathrm{H}_{3} \mathrm{P}_{4}$ resulted in efficient separation with reproducible peaks of L-citrulline and Larginine although all chromatograms showed stable baseline (Figure 3(c)). This finding is in agreement with Fekete et al. [22] who noted that $0.1 \% \mathrm{H}_{3} \mathrm{PO}_{4}$ acts as a good separation agent by increasing the polarity and improving the retention time of zwitterionic molecules including amino acids. Dolan [23] supported the notion that $0.1 \% \mathrm{H}_{3} \mathrm{P0}_{4}$ adequately provides reasonable buffering for amino acids separation by RP-HPLC. This showed that a concentration less than $1.0 \%$ $\mathrm{H}_{3} \mathrm{PO}_{4}$ as mobile phase provides efficient separation of amino acids, peptides, or proteins as demonstrated by Shibue et al. [24]. Thus, the mobile phase $0.1 \% \mathrm{H}_{3} \mathrm{PO}_{4}$ is proven to provide efficient separation and the best resolution of mixed standard, L-citrulline, and L-arginine.

The study also evaluated separation of mixed standard in two different columns, Zorbax Eclipse XDB- $\mathrm{C}_{18}$ and Gemini $\mathrm{C}_{18}$ using $0.1 \% \mathrm{H}_{3} \mathrm{P}_{4}$. Zorbax Eclipse $\mathrm{XDB}-\mathrm{C}_{18}$ did not provide good separation and resolution of L-citrulline and L-arginine as shown in Figure 4(a). A study by Barber and Joseph [25] showed that polar compounds were less separated and not well resolved using Zorbax Eclipse XDB$\mathrm{C}_{18}$ with a longer analysis time of 54 minutes. Efficient separation and resolution of L-citrulline and L-arginine from 


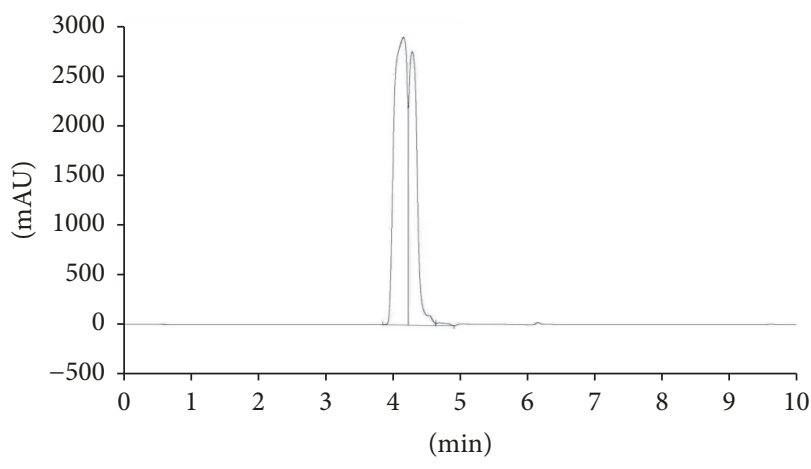

(a)

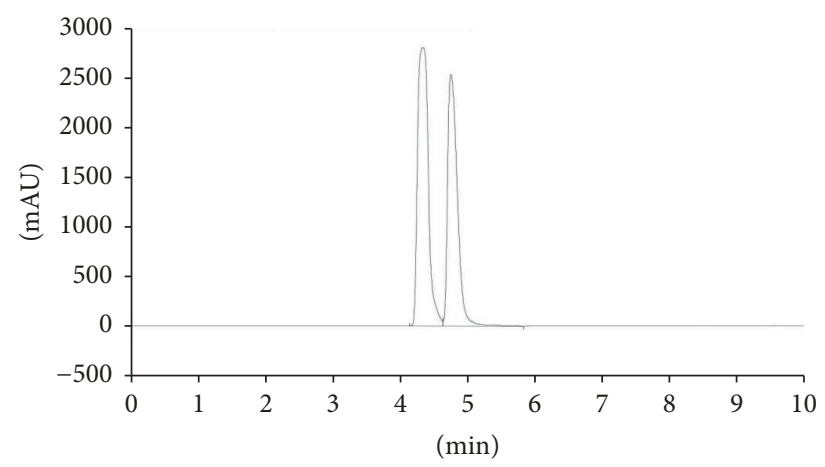

(b)

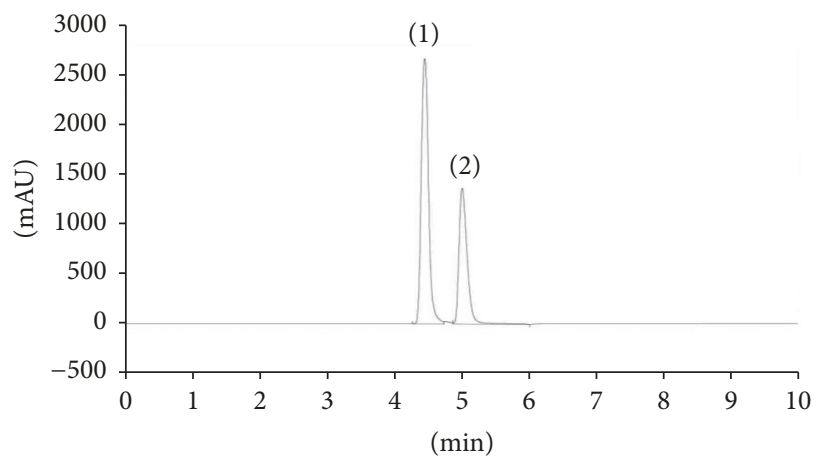

(c)

FIGURE 3: Comparative chromatograms showing isocratic RP-HPLC separation of mixed standard, L-citrulline, and L-arginine in different mobile phases: (a) $0.7 \% \mathrm{H}_{3} \mathrm{PO}_{4}$ : ACN (90:10); L-citrulline and L-arginine were unretained and coeluted at $k$ value close to zero; (b) $0.7 \%$ $\mathrm{H}_{3} \mathrm{PO}_{4}$; L-citrulline and L-arginine were slightly retained and partially separated; (c) $0.1 \% \mathrm{H}_{3} \mathrm{PO}_{4}$; L-citrulline and L-arginine were efficiently separated with reproducible peaks. The peaks marked represent (1) L-arginine and (2) L-citrulline.

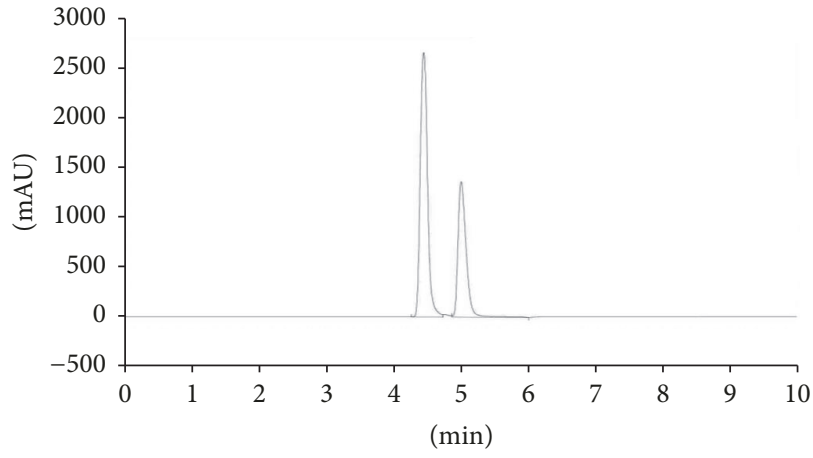

(a)

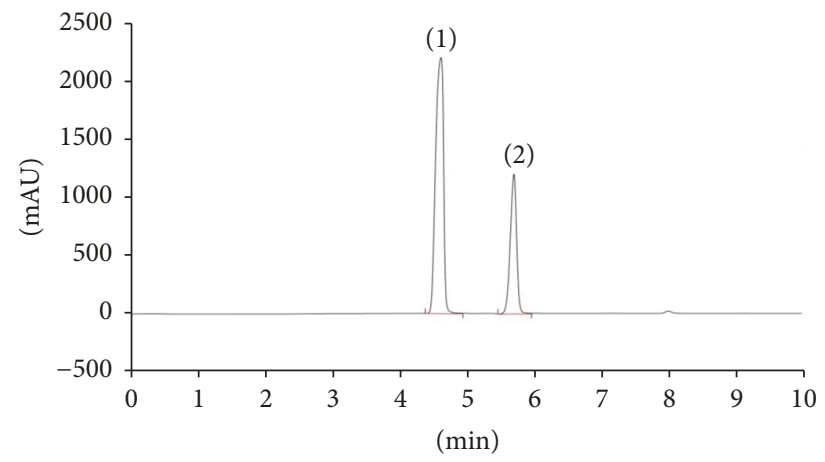

(b)

FIGURE 4: Comparative chromatograms showing isocratic RP-HPLC separation of mixed standard, L-citrulline, and L-arginine from 2 different columns: (a) Zorbax Eclipse XDB- $\mathrm{C}_{18}, 5 \mu \mathrm{m}$, and (b) Gemini $\mathrm{C}_{18}, 3 \mu \mathrm{m}$; efficient separation and the best resolution were achieved by the Gemini $\mathrm{C}_{18}$ column which showed that compounds are well separated. The peaks marked represent (1) L-arginine and (2) L-citrulline.

mixed standard were achieved using Gemini $\mathrm{C}_{18}$ as shown in Figure 4(b). L-citrulline and L-arginine are eluted at a short retention time with L-arginine, $4.773 \mathrm{~min}$, followed by L-citrulline at $5.787 \mathrm{~min}$ (Figure 5). Efficient separation of Lcitrulline with a retention time of about $4 \mathrm{~min}$ was achieved on the Gemini $\mathrm{C}_{18}$ column due to the high degree similarity of column with polar compounds [13]. Gemini $\mathrm{C}_{18}$ is a new generation hybrid column end-capped with porous silica as base core and polymer media coated on top of the silica core which exhibit silica-like mechanical properties of base material while similarly decreasing the number of residual silanols [26]. This result demonstrated that Gemini $\mathrm{C}_{18}$ is the most suited column for efficient separation of mixed standard, L-citrulline and L-arginine. 


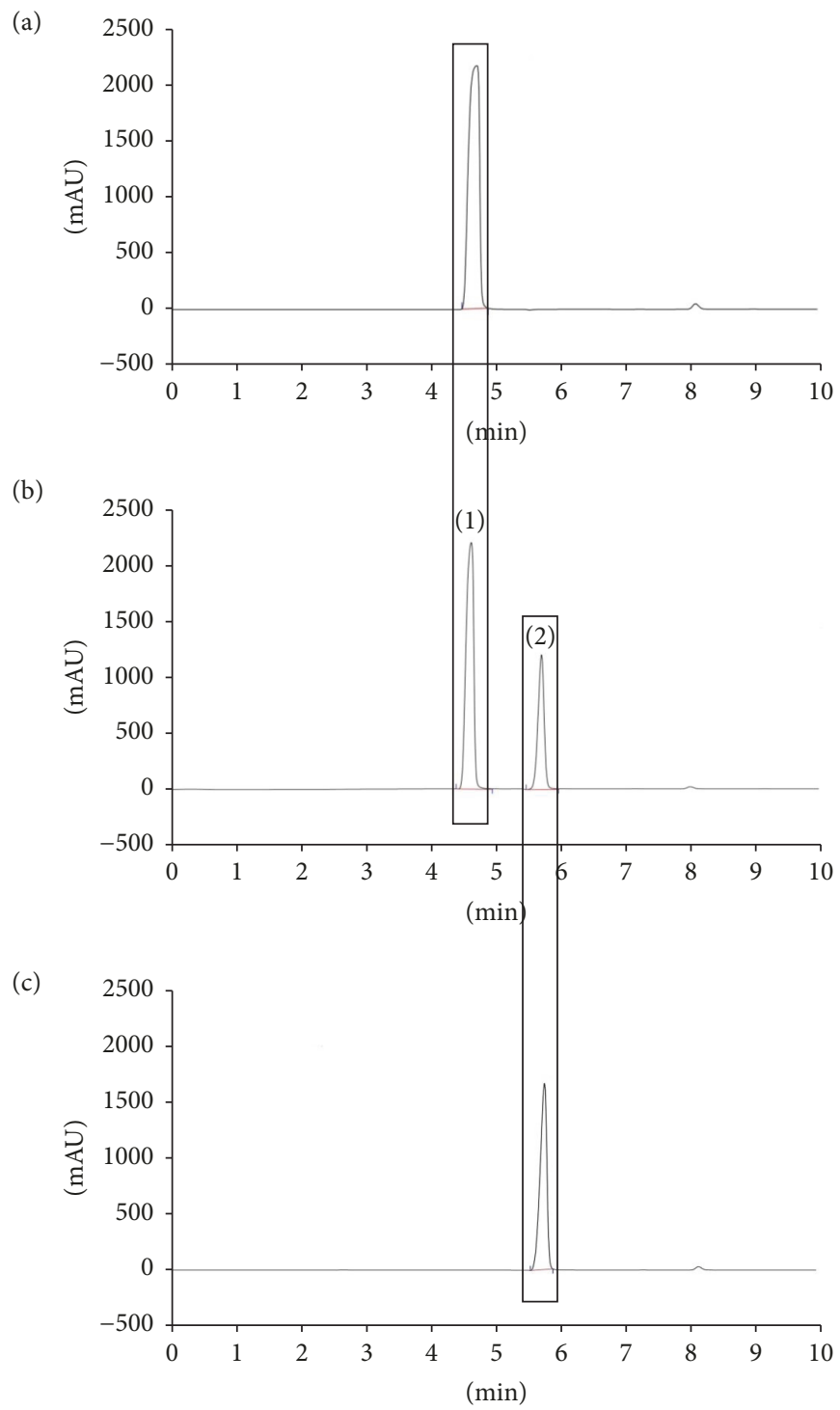

FIGURE 5: Comparative chromatograms showing isocratic RP-HPLC separation of individual and mixed standard, L-citrulline, and L-arginine using Gemini $\mathrm{C}_{18}$ : (a) L-arginine, (b) mixed standard, and (c) L-citrulline. The peaks marked represent (1) L-arginine and (2) L-citrulline.

The result from chromatography separation of Lcitrulline and L-arginine shown in Figure 5(b) demonstrated that the reverse-phase (RP) mode provided efficient separation and substantial retention achieved on both polar compounds without the need for derivatization. RP mode can efficiently be applied in this study although Brown et al. [27] proposed the use of hydrophilic interaction liquid chromatography (HILIC) mode after cyano- and pentafluorophenylpropyl stationary phases failed to retain target compounds including L-citrulline and L-arginine. HILIC mode is primarily used when separation of very polar compounds is needed or for incomplete chromatographic separation in RP mode [28]. However, HILIC mode required an expensive and robust system equipped with tandem mass spectrometric (MS) detection for L-citrulline and L-arginine separation in high biological matrix samples such as serum and plasma $[16,27]$. Complete chromatographic separation of L-citrulline and L-arginine by RP mode in this study eliminates the use of HILIC mode.

3.2. Validation of Isocratic RP-HPLC Method. Method validation of isocratic RP-HPLC method was performed by determination of linearity, LOD, LOQ, recovery, and intraday and interday analysis. The results of linearity, LOD, and LOQ were summarized in Table 2. Good linear regression equations of L-citrulline and L-arginine standard are displayed between corresponding peak areas versus concentrations of compounds based on the correlation coefficients $\left(R^{2}=\right.$ $0.9956, y=0.1664 x+2.4142$ and $R^{2}=0.9912, y=0.4100 x+$ 3.4850 , resp.). The LOD and LOQ for L-citrulline were $0.42 \mu \mathrm{g} / \mathrm{mL}$ and $1.28 \mu \mathrm{g} / \mathrm{mL}$ while those for L-arginine were $0.88 \mu \mathrm{g} / \mathrm{mL}$ and $2.66 \mu \mathrm{g} / \mathrm{mL}$, which demonstrated that isocratic RP-HPLC method was efficiently sensitive. The method had good accuracy that showed efficient recoveries for both 
TABLE 2: Calibration data of L-citrulline and L-arginine standard reported from isocratic RP-HPLC method.

\begin{tabular}{lccccc}
\hline Standard & $\begin{array}{c}\text { Concentration } \\
\text { range }(\mu \mathrm{g} / \mathrm{mL})\end{array}$ & Regression equation & $\begin{array}{c}\text { Correlation } \\
\text { coefficient } \\
\left(R^{2}\right)\end{array}$ & $\begin{array}{c}\text { Limit of } \\
\text { detection } \\
(\mu \mathrm{g} / \mathrm{mL})\end{array}$ & $\begin{array}{c}\text { Limit of } \\
\text { quantification } \\
(\mu \mathrm{g} / \mathrm{mL})\end{array}$ \\
\hline L-citrulline & $0.01-1000$ & $y=0.1664 x+2.4142$ & 0.9956 & 0.42 & 1.28 \\
L-arginine & $0.01-500$ & $y=0.4100 x+3.4850$ & 0.9912 & 0.88 & 2.66 \\
\hline
\end{tabular}

TABLE 3: Recovery of L-citrulline and L-arginine standard reported from isocratic RP-HPLC method.

\begin{tabular}{|c|c|c|c|c|}
\hline Compounds & Added concentration $(\mu \mathrm{g} / \mathrm{mL})$ & Measured concentration $(\mu \mathrm{g} / \mathrm{mL})$ & Recovery (\%) & RSD (\%) \\
\hline \multirow{3}{*}{ L-citrulline } & 100 & 101.94 & 101.94 & 1.70 \\
\hline & 60 & 61.24 & 102.07 & 1.46 \\
\hline & 30 & 31.02 & 103.38 & 1.00 \\
\hline \multirow{3}{*}{ L-arginine } & 100 & 99.87 & 99.87 & 1.96 \\
\hline & 60 & 59.33 & 98.88 & 1.76 \\
\hline & 30 & 31.02 & 103.41 & 1.17 \\
\hline
\end{tabular}

TABLE 4: Intraday and interday analysis of L-citrulline and L-arginine standard reported from isocratic RP-HPLC method.

\begin{tabular}{|c|c|c|c|c|c|}
\hline \multirow{2}{*}{ Compounds } & \multirow{2}{*}{ Concentration $(\mu \mathrm{g} / \mathrm{mL})$} & \multicolumn{2}{|c|}{ Intraday $(n=3)(\%)$} & \multicolumn{2}{|c|}{ Interday $(n=3)(\%)$} \\
\hline & & Mean & RSD & Mean & RSD \\
\hline \multirow{3}{*}{ L-citrulline } & 150 & 101.25 & 1.23 & 100.42 & 1.09 \\
\hline & 60 & 102.07 & 1.46 & 103.44 & 1.01 \\
\hline & 20 & 116.44 & 2.03 & 116.22 & 0.37 \\
\hline \multirow{3}{*}{ L-arginine } & 150 & 102.01 & 0.56 & 103.83 & 2.05 \\
\hline & 60 & 96.26 & 1.04 & 97.36 & 1.13 \\
\hline & 20 & 95.41 & 0.68 & 95.94 & 0.33 \\
\hline
\end{tabular}

compounds ranging from $98.88 \%$ to $103.41 \%$ (Table 3 ). The RSD (\%) for intraday and interday precision ranged from $0.37 \%$ to $1.09 \%$ for L-citrulline and $0.33 \%$ to $2.05 \%$ for Larginine, in which both $\mathrm{RSD} \leq 2 \%$ (Table 4 ). These validation results confirmed that the isocratic RP-HPLC method is precise, accurate, and sensitive for simultaneous quantification of L-citrulline and L-arginine.

3.3. Quantification of L-Citrulline and L-Arginine Contents in Two Different Watermelon Extracts. Consumption of watermelon extracts rich in $\mathrm{L}$-citrulline and $\mathrm{L}$-arginine is proven to be beneficial for diseases prevention. Thus, a rapid, reliable, and efficient isocratic RP-HPLC method is essential for simultaneous quantification of these amino acids in juice extracts and methanol extracts. The chromatographic profiles of both extracts in red watermelon and yellow crimson watermelon are presented in Figure 6. Quantification of Lcitrulline and L-arginine was performed using Chromeleon software. The content was calculated based on the calibration curve of L-citrulline and L-arginine standard achieved with good correlation coefficients and linear regression equations, $R^{2}=0.9956, y=0.1664 x+2.4142$ and $R^{2}=0.9912$, $y=0.4100 x+3.4850$, respectively. The results are tabulated in Table 5.

Red watermelon juice extract showed slightly high yield of L-citrulline in rind, $45.02 \mathrm{mg} / \mathrm{g}$ compared to flesh,
$43.81 \mathrm{mg} / \mathrm{g}$. Similar trends were shown in L-citrulline content in rind and flesh of yellow crimson juice extract, $16.61 \mathrm{mg} / \mathrm{g}$ and $15.77 \mathrm{mg} / \mathrm{g}$, respectively. This finding is in accordance with the study by Jayaprakasha et al. [13] which found that rinds from $C$. vulgaris watermelon varieties of petite treat and jamboree watermelon and yellow crimson watermelon contained significantly high L-citrulline, $13.95 \mathrm{mg} / \mathrm{g}$, $20.84 \mathrm{mg} / \mathrm{g}$, and $28.46 \mathrm{mg} / \mathrm{g}$, respectively, compared to flesh, $11.25 \mathrm{mg} / \mathrm{g}, 16.73 \mathrm{mg} / \mathrm{g}$, and $14.74 \mathrm{mg} / \mathrm{g}$, respectively, using RP-HPLC method. L-arginine content in red watermelon juice extract was higher in flesh, $11.10 \mathrm{mg} / \mathrm{g}$, compared to rind, $3.39 \mathrm{mg} / \mathrm{g}$. L-arginine content was approximately 3 fold lower than L-citrulline in red watermelon flesh juice extract. Consumption of watermelon flesh juice extract aided in efficient conversion of significantly high L-citrulline, a potent endogenous precursor to L-arginine in the kidney, which resulted in increased plasma L-arginine concentration. Findings by Collins et al. [29] proved that plasma L-arginine concentration increased by $95.2 \pm 3.5 \mu \mathrm{M}$ and $108.0 \pm 4.1 \mu \mathrm{M}$ compared to normal plasma baseline, $86.4 \pm 3.5 \mu \mathrm{M}$ after 3 weeks of consumption of $780 \mathrm{~mL}(\sim 1 \mathrm{~g} \mathrm{~L}$-citrulline/day) to $1560 \mathrm{~mL}(\sim 2 \mathrm{~g}$ L-citrulline/day) of watermelon juices. Recently, a study by Bailey et al. [30] supported the notion of the increased plasma L-arginine concentration by $116 \pm$ $9 \mu \mathrm{M}$ compared to placebo, $67 \pm 13 \mu \mathrm{M}$ after 2 weeks of $300 \mathrm{~mL} /$ day watermelon juice consumption, which contains 


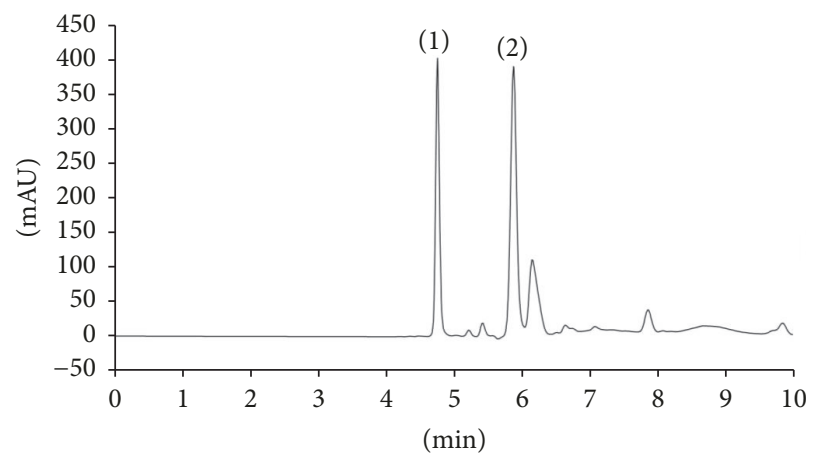

(a)

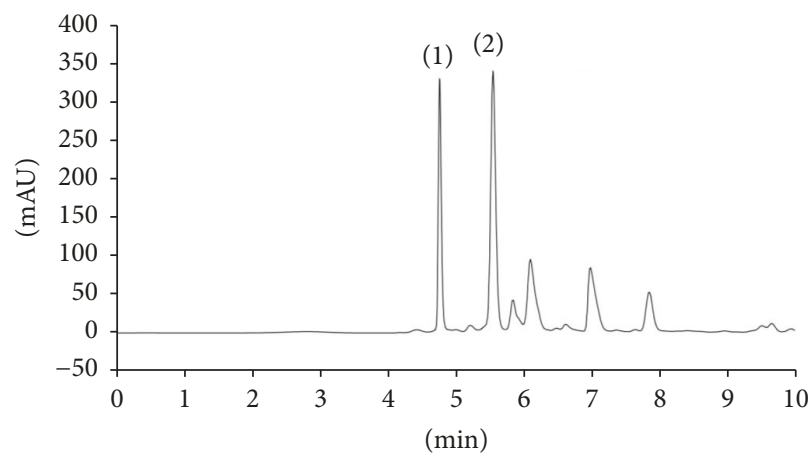

(c)

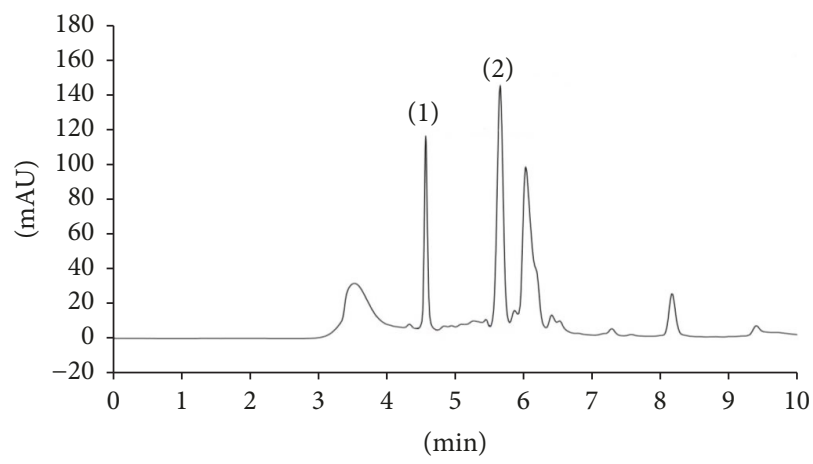

(e)

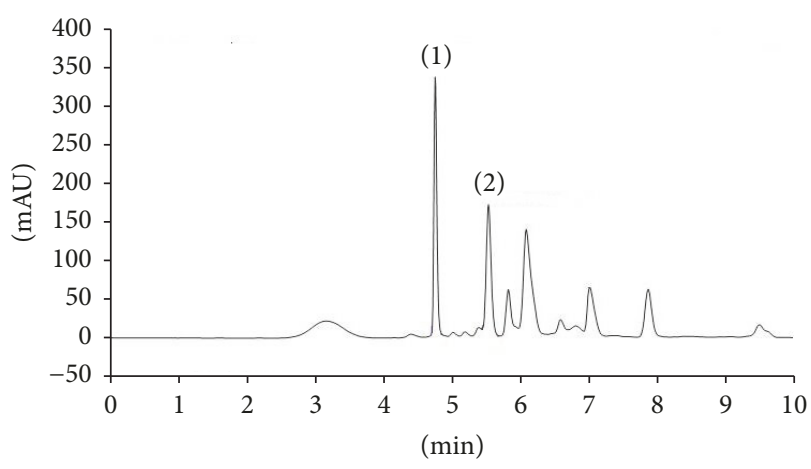

(g)

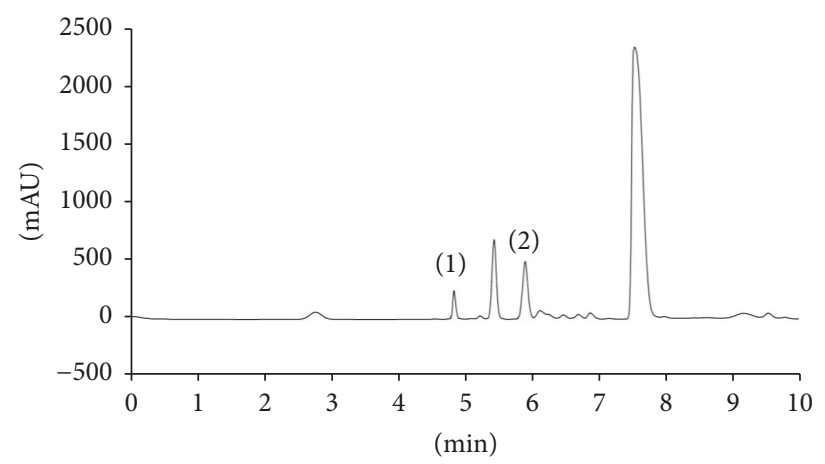

(b)

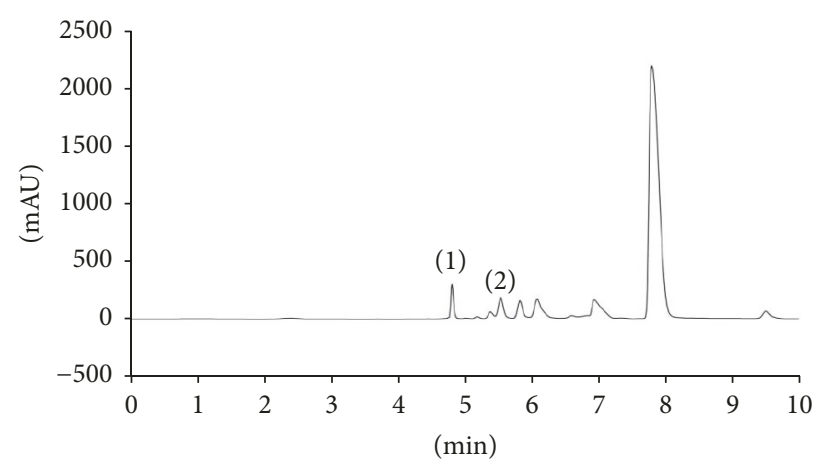

(d)

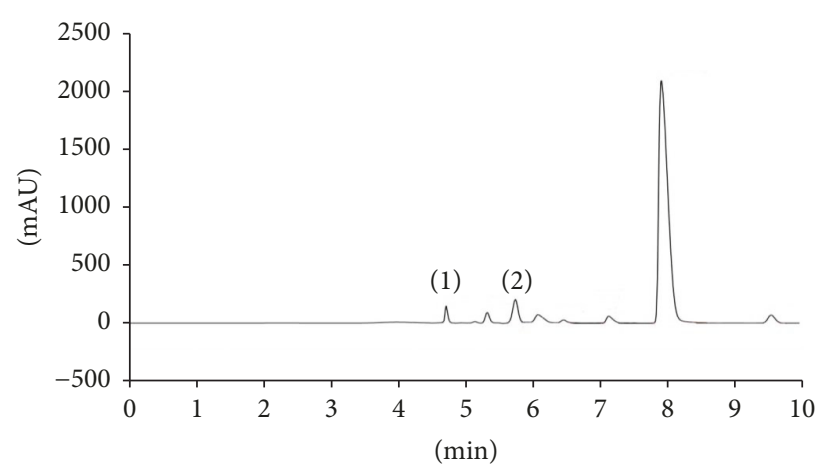

(f)

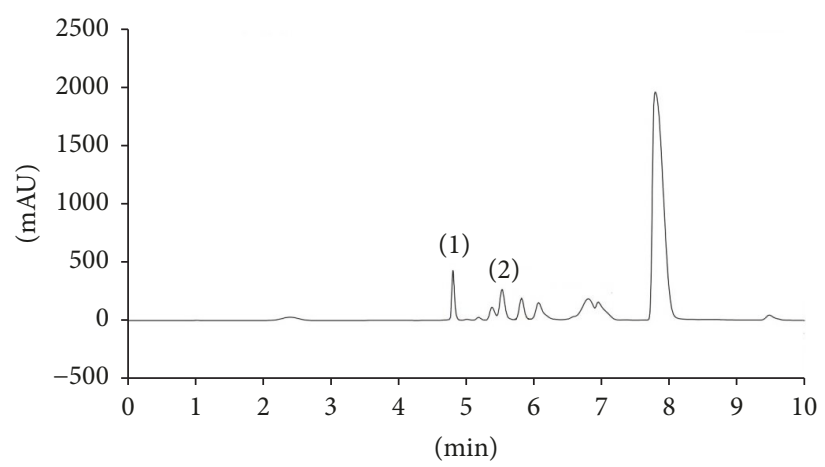

(h)

FIGURE 6: Comparative chromatographic profiles showing isocratic RP-HPLC separation of flesh and rind from juice extracts and methanol extracts of red watermelon and yellow crimson watermelon using Gemini $\mathrm{C}_{18}$. Red watermelon: (a) flesh juice extract, (b) rind juice extract, (c) crude flesh extract, and (d) crude rind extract. Yellow crimson watermelon: (e) flesh juice extract, (f) rind juice extract, (g) crude flesh extract, and (h) crude rind extract. The peaks marked represent (1) L-arginine and (2) L-citrulline. 
TABLE 5: L-citrulline and L-arginine contents ( $\mathrm{mg} / \mathrm{g})$ in juice extract and methanol extract of watermelon flesh and rind juices.

\begin{tabular}{lcccc}
\hline Watermelon & Compounds & Juices & Juice extract $(\mathrm{mg} / \mathrm{g})$ & Methanol extract $(\mathrm{mg} / \mathrm{g})$ \\
\hline \multirow{3}{*}{ Red } & L-citrulline & Flesh & 43.81 & 16.22 \\
& & Rind & 45.02 & 24.99 \\
& L-arginine & Flesh & 11.10 & 6.42 \\
& & Rind & 3.39 & 4.08 \\
Yellow crimson & \multirow{2}{*}{ L-citrulline } & Flesh & 15.77 & 13.91 \\
& & Rind & 16.61 & 16.03 \\
& L-arginine & Flesh & 8.23 & 6.68 \\
& & Rind & 11.14 & 8.41 \\
\hline
\end{tabular}

$\sim 3.4 \mathrm{~g}$ L-citrulline/day. L-citrulline content in crude flesh and rind extract of red watermelon and yellow crimson watermelon varied in the range of $13.91-24.99 \mathrm{mg} / \mathrm{g}$, while $\mathrm{L}$-arginine content was in the range of $4.08-8.41 \mathrm{mg} / \mathrm{g}$. Lcitrulline and L-arginine content is much lower in methanol extracts compared to juice extracts. Fish and Bruton [17] stated that methanol extracts may diminish the solubility of amino acids, thus marked reduction in amino acids yield. The quantitative results confirmed that watermelon juice extracts most effectively quantified higher yield of L-citrulline and Larginine, and this study's outcomes may possibly suggest that juice extraction method is best in optimizing amino acids yield.

\section{Conclusion}

The isocratic RP-HPLC method has been successfully developed for separation and quantification of L-citrulline and L-arginine content in both watermelon extracts of flesh and rind using the selected mobile phase $\left(0.1 \% \mathrm{H}_{3} \mathrm{PO}_{4}\right)$ in Gemini $\mathrm{C}_{18}$. The established isocratic RP-HPLC method provides evidence that $\mathrm{L}$-citrulline and $\mathrm{L}$-arginine are best retained using Gemini $\mathrm{C}_{18}$ column. The validated method is robust, sensitive, accurate, and precise with good linearity $\left(R^{2} \geq 0.99\right)$, low values of LOD and LOQ, recoveries within $98.88 \%-103.41 \%$, and \%RSD precision less than $2 \%$. Juice extract effectively yielded higher L-citrulline and Larginine content by juice extraction method; thus it is potentially used for quantitative amino acids analysis. The present study procedure may provide a basis for separation and quantification of L-citrulline and L-arginine in local watermelons. The high content of L-citrulline and L-arginine suggested watermelons as a good source of nutraceutical and health benefits ingredients. However, further researches are necessary to explore biological activities such as aphrodisiac properties of these active constituents in watermelons to support their potential in human diet and prevention of health related diseases.

\section{Conflicts of Interest}

The authors declare that there are no conflicts of interest that would prejudice the impartiality of this scientific work.

\section{Acknowledgments}

The authors gratefully acknowledge (1) the Ministry of Higher Education, Malaysia, through Fundamental Research Grant Scheme [FRGS Grant no. FRGS/1/2016/WAB01/UITM/02/3] and Institute of Research Management \& Innovation (IRMI), Universiti Teknologi MARA (UiTM), for funding the study; (2) Dr. Maizatul Hasyima Omar from Phytochemistry Unit, Institute for Medical Research, Kuala Lumpur, for providing analytical training; and (3) Atta-ur-Rahman Institute (AuRIns), UiTM Puncak Alam, Centre of Medical Laboratory Technology, and Centre of Postgraduate Study, Faculty of Health Sciences, UiTM, Selangor, Puncak Alam Campus, for providing facilities throughout this study.

\section{References}

[1] M. K. A. Mohammad, M. I. Mohamed, A. M. Zakaria, H. R. Abdul Razak, and W. M. M. Saad, "Watermelon (Citrullus lanatus (Thunb.) Matsum. and Nakai) juice modulates oxidative damage induced by low dose X-ray in mice," BioMed Research International, vol. 2014, Article ID 512834, 6 pages, 2014.

[2] G. K. Jayaprakasha and B. S. Patil, "A metabolomics approach to identify and quantify the phytochemicals in watermelons by quantitative ${ }^{1}$ HNMR," Talanta, vol. 153, pp. 268-277, 2016.

[3] A. Naz, M. S. Butt, M. T. Sultan, M. M. N. Qayyum, and R. S. Niaz, "Watermelon lycopene and allied health claims," Experimental and Clinical Sciences Journal, vol. 13, pp. 650-666, 2014.

[4] A. Figueroa, M. A. Sanchez-Gonzalez, P. M. Perkins-Veazie, and B. H. Arjmandi, "Effects of watermelon supplementation on aortic blood pressure and wave reflection in individuals with prehypertension: A pilot study," American Journal of Hypertension, vol. 24, no. 1, pp. 40-44, 2011.

[5] A. Poduri, D. L. Rateri, S. K. Saha, S. Saha, and A. Daugherty, "Citrullus lanatus 'sentinel' (watermelon) extract reduces atherosclerosis in LDL receptor-deficient mice," The Journal of Nutritional Biochemistry, vol. 24, no. 5, pp. 882-886, 2013.

[6] W. Liu, S. Zhao, Z. Cheng, X. Wan, Z. Yan, and S. R. King, "Lycopene and citrulline contents in watermelon (Citrullus lanatus) fruit with different ploidy and changes during fruit development," Acta Horticulturae, vol. 871, pp. 543-550, 2010.

[7] A. R. Davis, C. L. Webber III, W. W. Fish, T. C. Wehner, S. King, and P. Perkins-Veazie, "L-citrulline levels in watermelon cultigens tested in two environments," HortScience, vol. 46, no. 12, pp. 1572-1575, 2011. 
[8] A. Shafaei, A. F. Aisha, M. J. Siddiqui, and Z. Ismail, "Analysis of L-citrulline and L-arginine in Ficus deltoidea leaf extracts by reverse phase high performance liquid chromatography," Pharmacognosy Research, vol. 7, no. 1, pp. 32-37, 2015.

[9] E. Curis, I. Nicolis, C. Moinard et al., "Almost all about citrulline in mammals," Amino Acids, vol. 29, no. 3, pp. 177-205, 2005.

[10] M. J. Romero, D. H. Platt, R. B. Caldwell, and R. W. Caldwell, "Therapeutic use of citrulline in cardiovascular disease," Cardiovascular Drug Reviews, vol. 24, no. 3-4, pp. 275-290, 2006.

[11] G. Wu, J. K. Collins, P. Perkins-Veazie et al., "Dietary supplementation with watermelon pomace juice enhances arginine availability and ameliorates the metabolic syndrome in zucker diabetic fatty rats," Journal of Nutrition, vol. 137, no. 12, pp. 2680 2685, 2007.

[12] A. M. Rimando and P. M. Perkins-Veazie, "Determination of citrulline in watermelon rind," Journal of Chromatography A, vol. 1078, no. 1-2, pp. 196-200, 2005.

[13] G. K. Jayaprakasha, K. N. Chidambara Murthy, and B. S. Patil, "Rapid HPLC-UV method for quantification of L-citrulline in watermelon and its potential role on smooth muscle relaxation markers," Food Chemistry, vol. 127, no. 1, pp. 240-248, 2011.

[14] G. Wu and C. J. Meininger, "Analysis of Citrulline, Arginine, and Methylarginines Using High-Performance Liquid Chromatography," Methods in Enzymology, vol. 440, pp. 177-189, 2008.

[15] L. Wang, R. Xu, B. Hu et al., "Analysis of free amino acids in Chinese teas and flower of tea plant by high performance liquid chromatography combined with solid-phase extraction," Food Chemistry, vol. 123, no. 4, pp. 1259-1266, 2010.

[16] S. Shin, S.-M. Fung, S. Mohan, and H.-L. Fung, "Simultaneous bioanalysis of L-arginine, L-citrulline, and dimethylarginines by LC-MS/MS," Journal of Chromatography B, vol. 879, no. 7-8, pp. 467-474, 2011.

[17] W. W. Fish and B. D. Bruton, "Quantification of L-citrulline and other physiologic amino acids in watermelon and various cucurbits," Cucurbitaceae, vol. 2010, pp. 152-154, 2010.

[18] P. Markowski, I. Baranowska, and J. Baranowski, "Simultaneous determination of L-arginine and 12 molecules participating in its metabolic cycle by gradient RP-HPLC method. Application to human urine samples," Analytica Chimica Acta, vol. 605, no. 2, pp. 205-217, 2007.

[19] D. J. Dietzen, A. L. Weindel, M. O. Carayannopoulos et al., "Rapid comprehensive amino acid analysis by liquid chromatography/tandem mass spectrometry: Comparison to cation exchange with post-column ninhydrin detection," Rapid Communications in Mass Spectrometry, vol. 22, no. 22, pp. 3481-3488, 2008.

[20] M. Sadji, P. M. Perkins-Veazie, N. F. Ndiaye et al., "Enhanced L-citrulline in parboiled paddy rice with watermelon (Citrullus lanatus) juice for preventing sarcopenia: A preliminary study," African Journal of Food Science, vol. 9, no. 10, pp. 508-513, 2015.

[21] M. W. Dong, Modern HPLC for Practicing Scientists, John Wiley and Sons, 2006.

[22] S. Fekete, J.-L. Veuthey, and D. Guillarme, "New trends in reversed-phase liquid chromatographic separations of therapeutic peptides and proteins: Theory and applications," Journal of Pharmaceutical and Biomedical Analysis, vol. 69, pp. 9-27, 2012.

[23] J. Dolan, A Guide to HPLC and LC-MS Buffer Selection, ACE, 2009.

[24] M. Shibue, C. T. Mant, and R. S. Hodges, "Effect of anionic ion-pairing reagent hydrophobicity on selectivity of peptide separations by reversed-phase liquid chromatography," Journal of Chromatography A, vol. 1080, no. 1, pp. 68-75, 2005.

[25] W. E. Barber and M. Joseph, Eclipse XDB-CN Provides Excellent Selectivity and Resolution for Urea Pesticides, 2004, https://www .agilent.com/cs/library/applications/5989-0930EN_low.pdf.

[26] E. M. Borges, "Silica, hybrid silica, hydride silica and nonsilica stationary phases for liquid chromatography," Journal of Chromatographic Science (JCS), vol. 53, no. 4, pp. 580-597, 2015.

[27] C. M. Brown, J. O. Becker, P. M. Wise, and A. N. Hoofnagle, "Simultaneous determination of $6 \mathrm{~L}$-arginine metabolites in human and mouse plasma by using hydrophilic-interaction chromatography and electrospray tandem mass spectrometry," Clinical Chemistry, vol. 57, no. 5, pp. 701-709, 2011.

[28] B. Buszewski and S. Noga, "Hydrophilic interaction liquid chromatography (HILIC)-a powerful separation technique," Analytical and Bioanalytical Chemistry, vol. 402, no. 1, pp. 231247, 2012.

[29] J. K. Collins, G. Wu, P. Perkins-Veazie et al., "Watermelon consumption increases plasma arginine concentrations in adults," Nutrition Journal, vol. 23, no. 3, pp. 261-266, 2007.

[30] S. J. Bailey, J. R. Blackwell, E. Williams et al., "Two weeks of watermelon juice supplementation improves nitric oxide bioavailability but not endurance exercise performance in humans," Nitric Oxide: Biology and Chemistry, vol. 59, pp. 1020, 2016. 

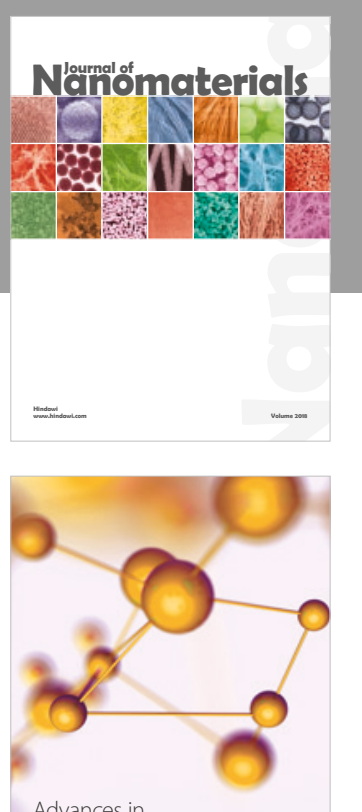

Physical Chemistry
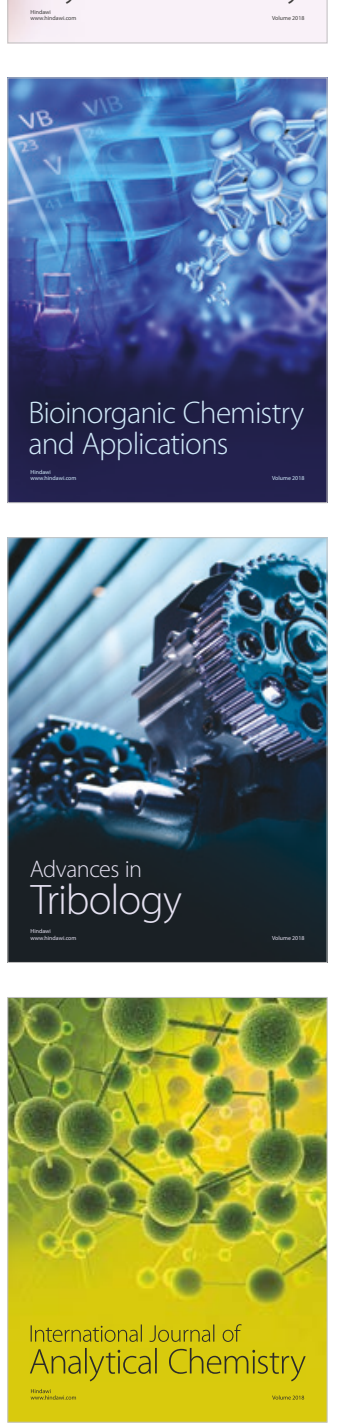

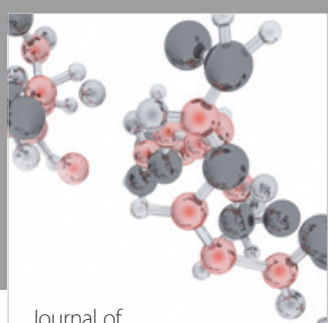

Analytical Methods

in Chemistry

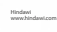

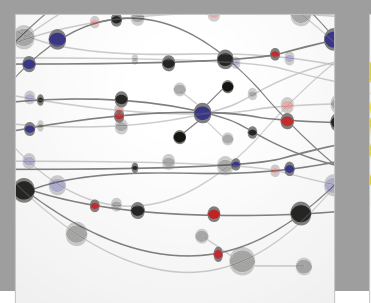

The Scientific World Journal

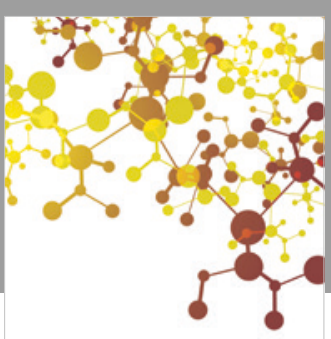

Journal of

Applied Chemistry
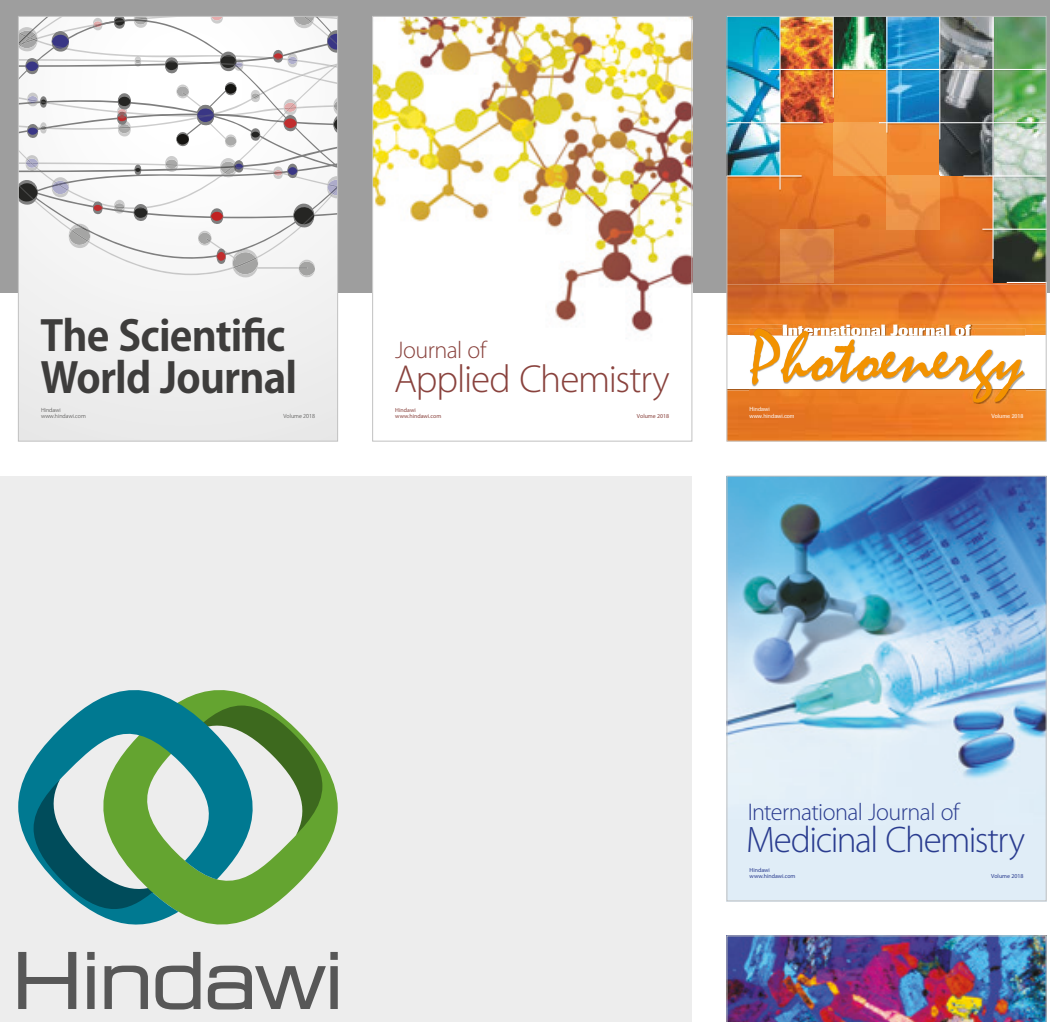

Submit your manuscripts at

www.hindawi.com
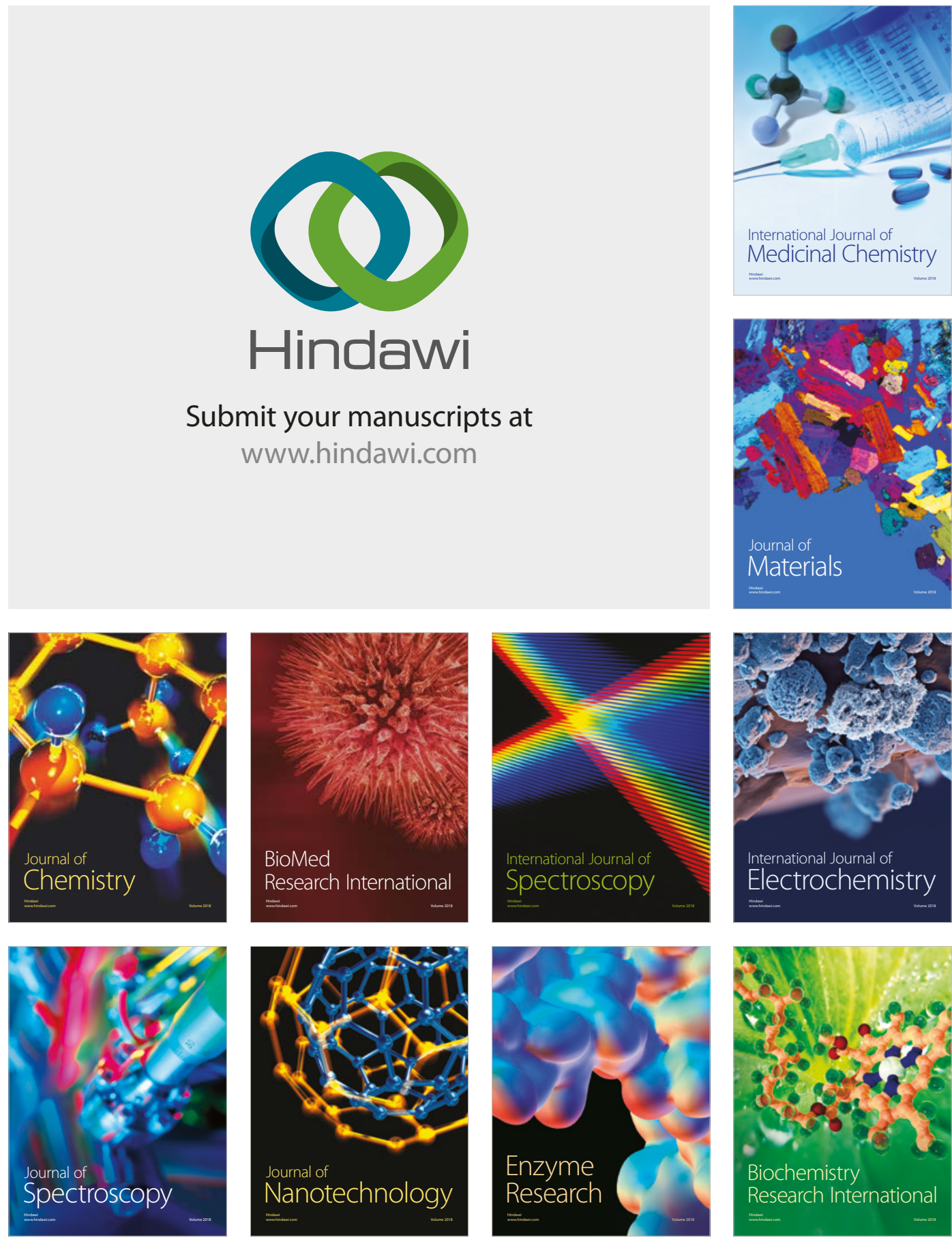
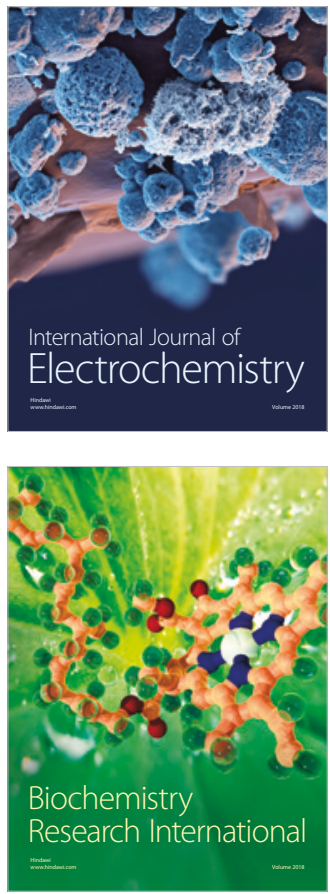